\title{
ON THE EFFECT OF A FOEHN ON COLD FRONTS IN THE VICINITY OF THE ALPS
}

\author{
(Research Note) \\ K. P. HOINKA \\ Institute of Atmospheric Physics, German Aerospace Research Establishment (DFVLR), \\ D-8031 Oberpfaffenhafen, F.R.G.
}

(Received in final form 15 April, 1987)

\begin{abstract}
The present note discusses physical mechanisms which may contribute to cold air channelling close to the Alps. This involves the modification of the prefrontal air by the warm foehn air and of the postfrontal air by blocking effects resulting in an increase in precipitation. Additionally the influence of a sloping surface in the vicinity of the orography is considered. The problems are discussed in term of a north-south-oriented cold front behaving as an atmospheric gravity current propagating along the east-west oriented Alps.
\end{abstract}

A significant number of summertime cold fronts in southeast Australia have gravitycurrent-like features (Garratt et al., 1985), including those occurring along the east coast of Australia referred to as the Southerly Buster. Similar types of cold fronts have been observed in the vicinity of the Rocky Mountains (Shapiro et al., 1985). In Europe, many cold fronts change their propagation speed significantly as soon as they approach the Alps with eastward-moving fronts being deformed, retarded or even diminished in intensity by the Alps. Some fronts propagate faster in the vicinity of the Alps than elsewhere (Figure 1). A rough estimate shows that close to the Alps the front in Figure 1 propagates at a speed of about $10 \mathrm{~m} \mathrm{~s}^{-1}$ (between 06 and 12 UTC) whereas north of the Danube river the front moves with about half this speed. Observations suggest that at least some of these fronts have the local character of a gravity-current, but not all claims to this effect seem to be well-founded.

A fochn is a warm, dry, and gusty wind descending in the lee of a mountain range. North of the Alps, in the prefrontal region, there frequently is a foehn which leads to less cloud formation in the lower troposphere than usually expected in prefrontal areas. The warm foehn air originating from the south-westerly regions replaces the rest of the cold air which has been transported towards the Alps. The foehn air is very dry up to 3 to $4 \mathrm{~km}$, and cannot support cloud formation or precipitation. This foehn effect can sometimes be observed up to a distance of $200 \mathrm{~km}$ north of the Alps, and is frequently noticed up to $50 \mathrm{~km}$ (Hoinka and Rosler, 1987). After the breakdown of the foehn, the polar air mass is advected towards the Alps behind the front and is blocked by the Alps, leading to frontal precipitation augmented by the orography, presumably because of uplift.

In this note, we show that frontal distortion through orographic channelling can occur under the influence of orography arising only from the thermodynamic effects of the 


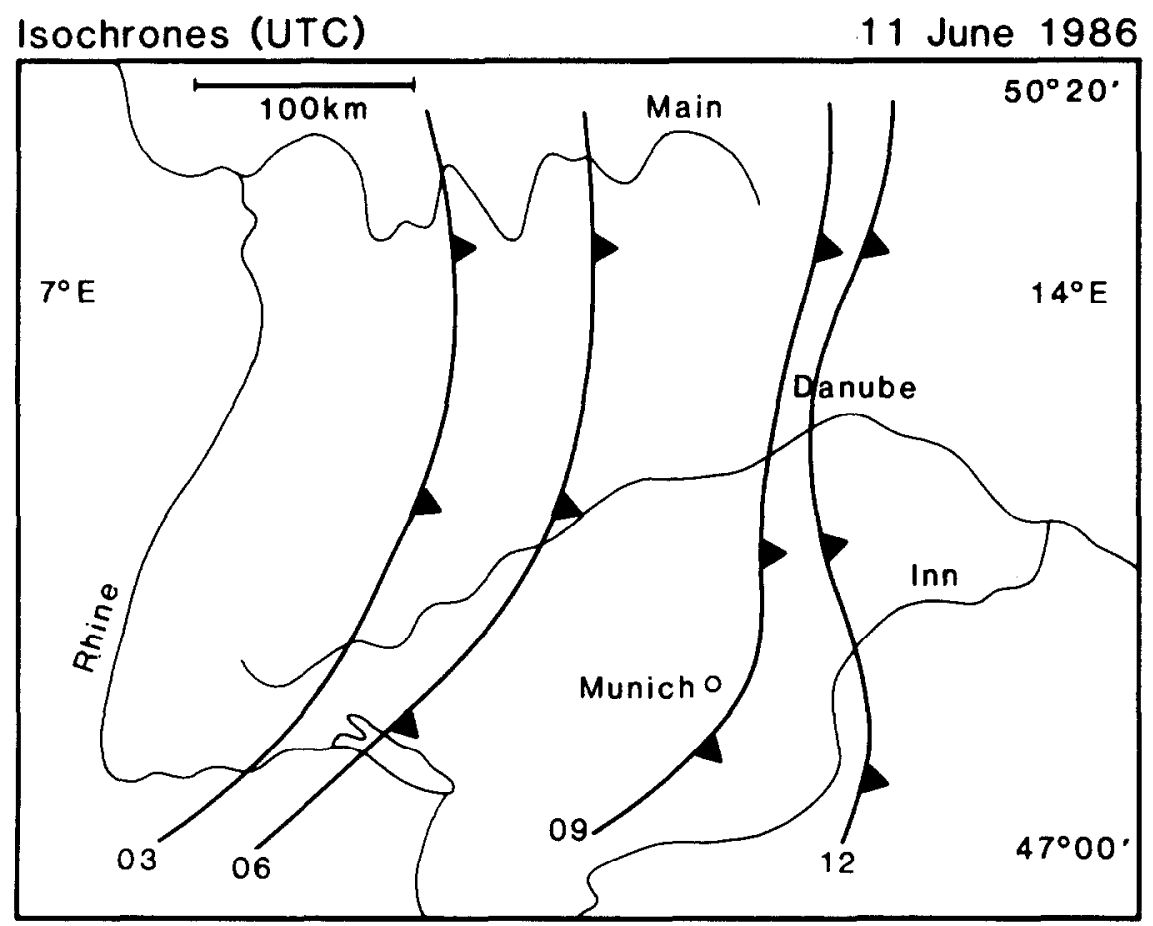

Fig. 1. Position of an observed cold front on June 11, 1986. The northern baseline of the Alps is along a line from south of the lake of Constance to about $60 \mathrm{~km}$ south of Munich.

orography on the flow, in particular from the low-level air-mass modification in both the prefrontal 'off-orography' flow (foehn) and the postfrontal 'on-orography' flow (blocking). We follow the analysis by Garratt (1985) very closely in which he discussed the boundary-layer effects on the cold air channelling at a coastline. The surface cold front is assumed to behave as an atmospheric gravity current, with speed ' $a$ ' given by

$$
a^{2}=\frac{g}{\Theta_{c}}\left(\Theta_{w}-\Theta_{c}\right) h,
$$

where $g$ is the acceleration due to gravity; $h$ is a representative depth of the cold air; and $\Theta_{w}$ and $\Theta_{c}$ are potential temperatures of the warm and cold air, respectively. Both air masses are assumed to be well mixed horizontally (parallel to the front) and vertically.

Observations suggest that between the warm foehn air and the colder surface air, there is a strong inversion at a height $z=\delta$ (Hoinka and Rosler, 1987). This $\delta$ increases northward with decreasing foehn effects at a rate of about $500 \mathrm{~m}$ per $50 \mathrm{~km}$. For present purposes, we represent this structure with a two-layer model (Figure 2): the lower layer of depth $\delta$ and temperature $\Theta_{1}$; and the foehn layer of temperature $\Theta_{F}$. For the transition 


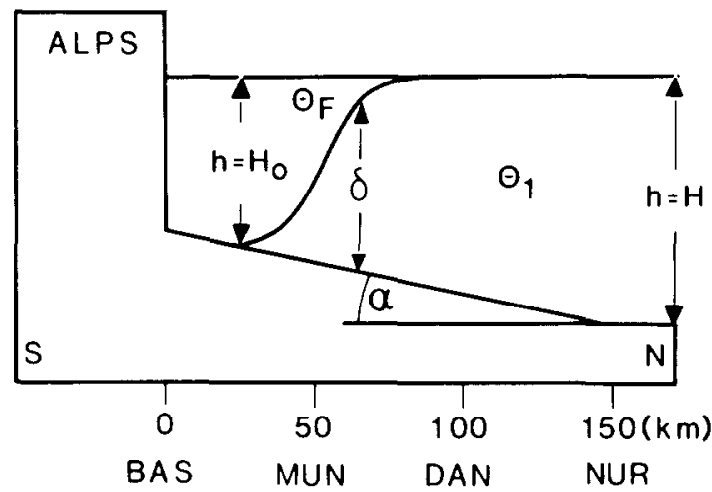

Fig. 2. Schematic picture of the two-layer model with a prefrontal foehn north of the Alps. The abbreviations stand for: baseline of the Alps (BAS), Munich (MUN), river Danube (DAN), and Nuremberg (NUR).

zone where $\delta<h$, we evaluate $\delta$ by using

$$
\delta(x)=H_{0}(1+\sin \phi) / 2+\left(h(x)-H_{0}\right),
$$

where $-\pi / 2 \leq \phi \leq+\pi / 2$. This expression for $\delta(x)$ is a simple analytical form of the observed interface. The mean prefrontal temperature $\Theta_{w}$ between the surface and $z=h$, is given simply (Garratt, 1986) as a weighted mean of the temperatures of the lower layer $\Theta_{1}$ and of the foehn layer $\Theta_{F}$

$$
\Theta_{w}=\frac{(h-\delta) \Theta_{F}}{h}+\frac{\delta \Theta_{1}}{h} .
$$

In case of flow towards the Alps, precipitation is generated by uplift, so that the heat of condensation produces warming of the air mass. To estimate the complex 'thermodynamic' effect of blocking, we consider for simplicity two extreme cases, the 'wellmixed' one and the 'evaporation' one. In a vertically well-mixed atmosphere in conjunction with a low cloud base, the heat of condensation is distributed in the layer between the ground and the cloud top, so that the postfrontal air is warmer than without the condensation. In the case of vertically stable stratification, parts of the rain evaporate in the sub-cloud layer, so that the air temperature decreases in this layer. The flow towards the Alps provides sufficient humidity so that the decrease in temperature continues until the air is close to saturation.

First let us consider an example for the 'well-mixed' case. An air mass at $290 \mathrm{~K}$ and mixing ratio of $5 \mathrm{~g} \mathrm{~kg}^{-1}$ is heated by about $(\Delta \Theta)_{M}=+1.5 \mathrm{~K}$ due to condensation as soon as the mixing ratio is reduced by $10 \%$ and the condensed water falls out as precipitation. For the 'evaporation' case, we assume an increase in relative humidity from 80 to $95 \%$ for the layer where the rain evaporates. Applying

$$
c_{p} \Delta T \cong L_{v} \Delta m
$$


with $c_{p}=10^{3} \mathrm{~J} /(\mathrm{kg} \mathrm{K}), L_{v}=2.5 \times 10^{6} \mathrm{~J} \mathrm{~kg}^{-1}$ and $\Delta m$ the difference in mixing ratios corresponding to the difference in relative humidities, leads to a decrease in temperature of about $(\Delta \Theta)_{E}=-5 \mathrm{~K}$.

The thermodynamic effect of blocking on the air might be roughly approximated by increasing or decreasing the postfrontal air by a heat excess due to condensation or evaporation. For the present purpose, we assume that this thermodynamic process is effective only in the first $100 \mathrm{~km}$ north of the Alps. We use typical values observed during strong foehn events with $\Theta_{F}=300 \mathrm{~K}, \Theta_{1}=295 \mathrm{~K}, \Theta_{c}=290 \mathrm{~K},(\Delta \Theta)_{E}=-5 \mathrm{~K}$ and $(\Delta \Theta)_{M}=1.5 \mathrm{~K}$. The depth of the fluid $h$ at the northernmost point is choosen to be $H=1000 \mathrm{~m}$. Qualitatively, the main conclusions of this note are unchanged if we choose different values for $H, \Theta_{F}, \Theta_{1}, \Theta_{c},(\Delta \Theta)_{M},(\Delta \Theta)_{E}$, and $\alpha$.

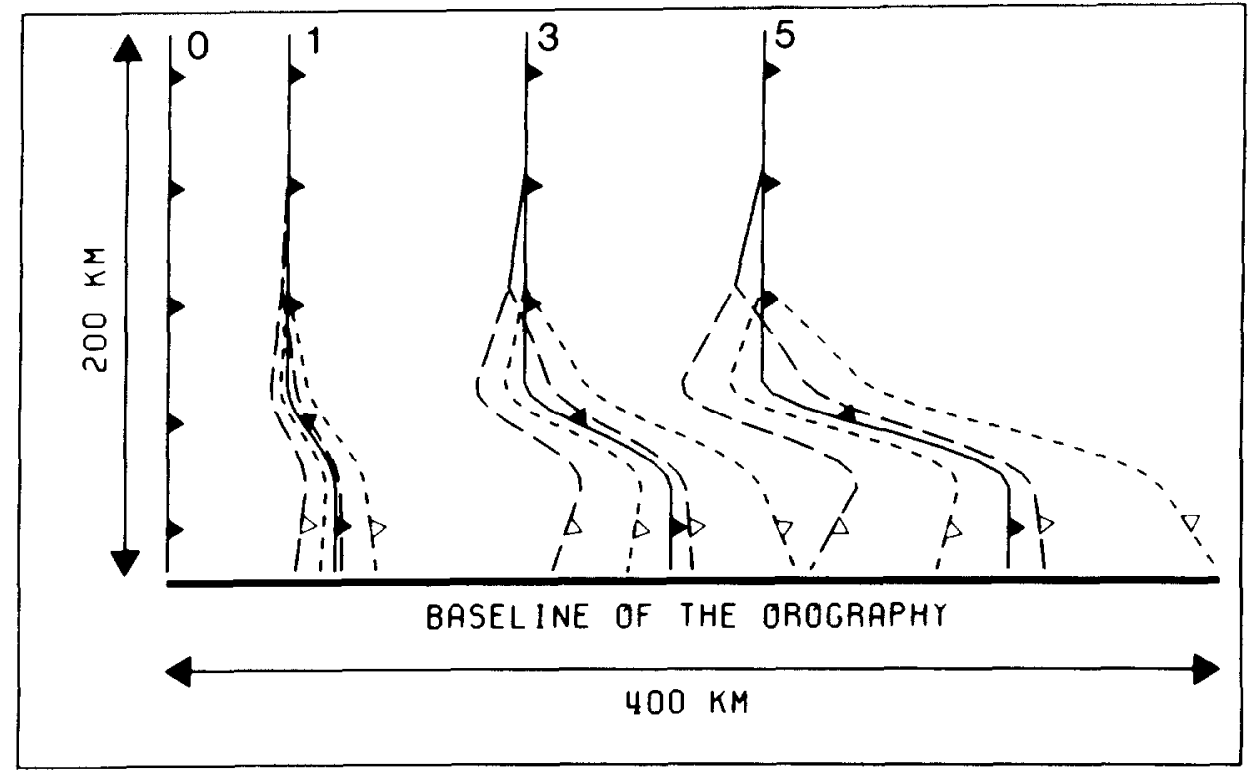

Fig. 3. Schematic representation of the frontal distortion along a east-west mountain arising from air mass modification due to a prefrontal foehn (full lines) and also due to postfrontal blocking effects (short-broken lines). The influence of sloping terrain is shown by the long-broken line. The position of the gravity-current leading edge (front) is shown at hours as indicated. The frontal positions for the 'well-mixed' ('evaporation') case are shown by the fronts on the left-hand side (right-hand side) of the frontal position corresponding to foehn (full lines). The following values are used: $\Theta_{F}=300 \mathrm{~K}, \Theta_{1}=295 \mathrm{~K}, \Theta_{c}=290 \mathrm{~K},(\Delta \Theta)_{M}=1.5 \mathrm{~K}$, $(\Delta \Theta)_{E}=-5 \mathrm{~K}, H=1000 \mathrm{~m}, \alpha=100 \mathrm{~m} / 50 \mathrm{~km}$.

In the first experiment, we assume that there is a prefrontal foehn but no postfrontal blocking and the slope of the terrain is zero $(\alpha=0)$. The results of the spatial variation in $\left(\Theta_{w}-\Theta_{c}\right)$ and, hence, in the speed $a$, is to produce sequential frontal positions as shown in Figure 3 (full lines). There is a strong acceleration of the cold air close to the mountains. The frontal speed at the northernmost point is $12.6 \mathrm{~m} \mathrm{~s}^{-1}$ and at the baseline of the Alps $17.8 \mathrm{~m} \mathrm{~s}^{-1}$. 
The thermodynamic effect of blocking in the 'mixing' case reduces the speed of the propagation to about $16.3 \mathrm{~m} \mathrm{~s}^{-1}$ in the vicinity of the mountain (Figure 3, short-broken lines). The next experiment shows the influence of a sloping lower boundary (Figure 3, long-broken lines). A realistic value for $\alpha$ is a slope of $100 \mathrm{~m}$ per $50 \mathrm{~km}$ northwards in southern Germany. The front is again decelerated at the baseline of the Alps. The speed is now $13.6 \mathrm{~m} \mathrm{~s}^{-1}$. In Figure 3 the frontal disortion is also shown for the 'evaporation' case. At the baseline of the Alps, the speed of propagation increases up to $22.2 \mathrm{~m} \mathrm{~s}^{-1}$ (short-broken lines). Besides this acceleration, it is observed that frontogenetic effects are strengthened by cooling through evaporation of falling rain in cloudy areas (Kurz, 1984). The effect of the sloping terrain reduces the propagation speed to $18.6 \mathrm{~m} \mathrm{~s}^{-1}$.

The results of this note corroborate some observed features of fronts in the vicinity of the Alps. However, real fronts are much more complicated. Other, nondiabatic processes, e.g., heating by increased incoming solar radiation due to less cloudiness in foehn areas, would also influence the propagation of density-current-type fronts. Additionally, actual fronts would be influenced by differential friction and would be influenced dynamically by orography (e.g., modification of the prefrontal low-level jet) in addition to the above mechanisms.

\section{References}

Garratt, J. R.: 1986, 'Boundary-Layer Effects on Cold Fronts at a Coastline', Boundary-Layer Meteorol. 36, 101-105.

Garratt, J. R., Physick, W. L., Smith, R. K., and Troop, A. J.: 1985, 'The Australian Summertime Cool Change II: Mesoscale Aspects', Mon. Weather Rev. 113, 202-223.

Hoinka, K. P. and Rösler, F.: 1987, 'The Surface Layer on the Leeside of the Alps During Foehn', Met. Atmosph. Phys. (in press).

Kurz, M.: 1984, 'About the Influence of Nondiabatic Processes on Frontogenesis Near the Ground', in Int. Symp. on Nowcasting, 2nd, Norrkoping, Sweden, September, 3-7, 1984, Nowcasting II: Mesoscale Observations and Very-Short-Range Forcasting: Proceedings, pp. 63-67.

Shapiro, M. A., Hampel, T., Rotzoll, D., and Mosher, F.: 1985, 'The Frontal Hydraulic Head: A Micro- $\alpha$ Scale $(-1 \mathrm{~km})$ Triggering Mechanism for Mesoconvective Weather Systems', Mon. Weather Rev. 113, $1166-1183$. 